\section{Naive realism in public perceptions of neuroimages}

\section{Madeleine Keehner and Martin H. Fischer}

The Perspectives article by Illes et al. (Neurotalk: improving the communication of neuroscience research, Nature Reviews Neuroscience 11, 61-69 (2010)) ${ }^{1}$ discussed the thorny but important issue of communicating neuroscience research. The authors identified a number of challenges for neuroscientists seeking to present their often complex findings in an accessible way. Among their specific recommendations was the need for more empirical research on the communication of neuroscience to the general public.

One emerging issue in this field is the 'dazzle effect' of brain images in scientific reports. It seems that merely including a picture of a brain increases the perceived credibility of research findings in the eyes of non-experts ${ }^{2}$. We explored this phenomenon to establish exactly what properties of brain images make them so seductive ${ }^{3}$. Our findings suggest that the more concrete and 'brain-like' the image is, the more credibility it has. Specifically, brain images that appeared highly three-dimensional and object-like (as rated by novices in a separate study) were more likely to convince naive readers that an accompanying written report contained sound scientific reasoning. By contrast, more abstract, schematic neuroscience formats resulted in lower credibility ratings for accompanying texts.

Why should brain images that resemble solid, tangible objects make people believe in research findings more strongly? In the neuroethics literature it has been claimed that novices tacitly view neuroimages as analogue representations of events in the brain, rather like photographs, produced by a kind of 'brain camera's . Thus, it is possible that the more object-like images were viewed not as graphics (technical visualizations that represent information in a non-direct, schematic way) but as depictions (images that directly capture real, visible objects and events, and do not require technical conventions or expert schemas for interpretation) ${ }^{5}$. Such apparent (but not actual) directness leads to increased feelings of fluency ${ }^{6-8}$ (the belief that information is easy to extract and understand), which in turn makes information more likely to be judged as true ${ }^{9}$.

Findings such as these reveal something important about public perceptions of neuroscience images - the appearance of threedimensionality or tangibility in presentation formats increases the perceived validity of associated findings. This bias is consistent with previous research on user perceptions of graphics: three-dimensional renderings are not only preferred but also assumed by non-experts to be more 'scientific' and easier to understand than schematic two-dimensional displays ${ }^{10,11}$, presumably because viewing them seems more similar to direct perception of objects in the real world. This misplaced faith in realistic, object-like displays has been termed 'naive realism' ${ }^{12}$.

With advances in visualization techniques, it is becoming possible to produce increasingly photorealistic, three-dimensional brain images to represent neuroscientific findings. Although these tools allow us to explore and visualize data in new and exciting ways, it is important to understand the effects of such compelling graphics on public perceptions of the results ${ }^{13,14}$. If a picture is truly worth a thousand words, the images we select to present our findings might be saying more than we think.

Madeleine Keehner is at the School of Psychology, University of Dundee, Dundee DD1 4HN, UK Correspondence to M.K. e-mail:m.m.keehner@dundee.ac.uk doi: 10.1038/nrn2773-c1 Published online 12 January 2011

1. Illes, J. et al. Neurotalk: improving the communication of neuroscience research. Nature Rev. Neurosci. 11, 61-69 (2010).

2. McCabe, D. P. \& Castel, A. D. Seeing is believing: the effect of brain images on judgments of scientific reasoning. Cognition, 107, 343-352 (2008).

3. Keehner, M., Mayberry, L. \& Fischer, M. H. Different clues from different views: the role of image format in public perceptions of neuroimaging results. Psychonomic Bull. Rev. (in press).

4. Roskies, A. L. Neuroimaging and inferential distance. Neuroethics 1,19-30 (2008).

5. Tversky, B. in Spatial Schemas and Abstract Thought (ed. Gattis, M.) 79-112 (MIT Press, Cambridge, 2001).

6. Schwarz, N. Metacognitive experiences in consumer judgment and decisionmaking. J. Consum. Psychol. 14, 332-348 (2004)

7. Schwarz, N., Sanna, L.J., Skurnik, I. \& Yoon, C. Metacognitive experiences and the intricacies of putting people straight: implications for debiasing and public information campaigns. Adv. Exp. Soc. Psychol. 39, 127-161 (2007).

8. Trout, J.D. Seduction without cause: uncovering explanatory neurophilia. Trends Cogn. Sci. 12, 281-282 (2008)

9. Reber, R. \& Schwarz, N. Effects of perceptual fluency on judgments of truth. Conscious Cogn. 8, 338-342 (1999).

10. Stewart, B. M., Cipolla, J. M. \& Best, L. A. in Readings in Education and Technology: proceedings of ICICTE 2008 (ed. Fernstrom, K.) 30-41 (University of the Fraser Valley Press, British Columbia, 2008).

11. Fischer, M. H. Do irrelevant depth cues affect the comprehension of bar graphs? Appl. Cogn. Psychol. 14, 151-162 (2000)

12. Smallman, H. S. \& St John, M. in Ergonomics in Design, 13, 2-13 (2005).

13. Farah, M. J. A picture is worth a thousand dollars. J. Cogn. Neurosci. 21, 623-624 (2009).

14. Morein-Zamir, S. \& Sahakian, B. J. Neuroethics and public engagement training needed for neuroscientists. Trends Cogn. Sci. 14, 49-51 (2010). 